\title{
Vértigo: revisión de los principales trastornos periféricos y centrales
}

\author{
Vertigo: review of the main peripheral and central disorders
}

\author{
Niza Harari Masri, ${ }^{*}$ Francisco Roa Castro*
}

\begin{abstract}
RESUMEN
El vértigo es un síntoma extremadamente común; éste puede llegar a ser incapacitante y a disminuir la calidad de vida. Es un término inespecífico que engloba varios síntomas: mareo, visión borrosa, inestabilidad, desequilibrio, entre otros. Constituye una de las causas más comunes de consulta otorrinolaringológica, neurológica y geriátrica. El vértigo es un síntoma subjetivo, difícil de evaluar o precisar, y su diagnóstico y tratamiento resulta problemático. La historia clínica y exploración física son elementos críticos para determinar el manejo en estos pacientes. Los signos y síntomas se sobreponen, entre las posibles etiologías, a otras patologías potencialmente mortales y, a su vez, pueden sobreponerse por otras patologías. Los estudios de escrutinio resultan poco específicos. El objetivo de este artículo es revisar las principales patologías de origen periférico y central.
\end{abstract}

Palabras clave: Vértigo, mareo, sistema vestibular, nistagmo.

Nivel de evidencia: III

\begin{abstract}
Vertigo is an extremely common symptom; it can become disabling and diminishes the quality of life. It is a nonspecific term that includes several symptoms from dizziness, blurred vision, instability, imbalance, among others. The vertigo it is one of the most common causes of otorhinolaryngological, neurological and geriatric consultation. It is a subjective symptom difficult to evaluate and to specify its diagnosis and treatment is problematic. The clinical history and physical examination are critical elements to determine the management in these patients. The signs and symptoms are superimposed among the possible etiologies, potentially deadly pathologies can be overcome by other pathologies; and the scrutiny studies are not very specific. The objective of this article is to review the main pathologies of peripheral and central origin.
\end{abstract}

Keywords: Vertigo, dizziness, vestibular system, nystagmus.

Level of evidence: III
* Otorrinolaringología y Cirugía de Cabeza y Cuello. Centro Médico ABC. Ciudad de México, México.

Recibido para publicación: 26/06/2019. Aceptado: 15/11/2019.

Correspondencia: Dra. Niza Harari Masri

Antiguo Camino a Tecamachalco Núm. 685-601, Col. Lomas del Olivo, Huixquilucan, 52788, Estado de México.

Teléfono: 6278-6643

E-mail: nizaharari@gmail.com

\footnotetext{
Abreviaturas:

VPPB = Vértigo postural paroxístico benigno.

$\mathrm{RM}=$ Resonancia magnética
}

Este artículo puede ser consultado en versión completa en: www.medigraphic.com/analesmedicos

\section{INTRODUCCIÓN}

Las definiciones de vértigo y mareo son generalmente muy vagas e inconsistentes. ${ }^{1-3}$ El Comité de Clasificación de Desórdenes Vestibulares ${ }^{4}$ propuso una categorización que, de acuerdo con esta clasificación, describe al vértigo como una falsa sensación (ilusión) de movimiento o movimiento distorsionado de uno, aun cuando el movimiento es normal, «las cosas giran». ${ }^{5,6}$ En contraste, el mareo es la sensación de desorientación en el espacio sin sensación de movimiento anormal; ${ }^{2}$ el desequilibrio se define como la sensación tambaleante o fuera de balance; el presíncope se define como la sensación de perder la consciencia o el conocimiento, y finalmente, el aturdimiento se refiere a aquellos síntomas vagales, o bien a una sensación de desconexión con el medio que lo rodea. ${ }^{1,7}$ 
Ya que los síntomas son subjetivos, es importante describir sus características, su inicio, gatillos y su duración. Hay que determinar si es un episodio único y agudo, constante o recurrente, qué factores son los que lo agravan o disminuyen o cualquier otro síntoma asociado, sean síntomas otológicos o neurológicos (alteraciones de la conciencia, diplopía, disartria, oftalmoplejía, hemiparesia, parálisis facial) (Tablas 1 y 2). ${ }^{1,7}$ Asimismo, el diagnóstico diferencial del vértigo incluye causas de origen vestibular y central.

\section{Epidemiología}

El mareo y el vértigo son responsables de 5\% de consultas en el Servicio de Urgencias, ${ }^{2}$ y el mareo es el tercer síntoma más común en la práctica médica en general. En Estados Unidos, 10 millones de personas anualmente se presentan a consulta externa con vértigo o mareo, y una cuarta parte es admitida en el Servicio de Urgencias. ${ }^{8}$

De 0.7 a $3.2 \%$ de los eventos vasculares cerebrales debutan como un síndrome vestibular agudo en el
Servicio de Urgencias. Por este motivo, por una parte, una falla en el diagnóstico tiene un gran impacto en la calidad del paciente y, por la otra, contar con un diagnóstico acertado podría mejorar el pronóstico de los pacientes. ${ }^{9}$

\section{Sistema vestibular}

El sistema vestibular está categorizado por sus componentes centrales y periféricos. El sistema periférico está compuesto, de forma bilateral, por tres canales semicirculares (posterior, superior y lateral), sáculo y utrículo. Los canales semicirculares detectan los movimientos de rotación y angulares de la cabeza, mientas el utrículo y el sáculo responden a movimientos de aceleración lineal y gravedad, respectivamente. La información del sistema periférico, junto con la propiocepción y la vista, es procesada en el núcleo vestibular (sistema central) y es aquél que nos da la sensación de posición y balance. ${ }^{10}$

El síndrome vestibular agudo se define como un síndrome con vértigo o mareo continuo, acompañado de náusea o vómito, intolerancia al movimiento ce-

Tabla 1: Características clínicas de las principales causas de vértigo.

\begin{tabular}{|c|c|c|c|}
\hline & Desencadenante & Duración & Signos/síntomas asociados \\
\hline \multicolumn{4}{|l|}{ Periféricas } \\
\hline Neuritis vestibular & Viral & $\begin{array}{l}\text { Crisis vertiginosa } \\
\text { Más de } 24 \text { horas }\end{array}$ & $\begin{array}{l}\text { Neurovegetativos } \\
\text { No signos auditivos }\end{array}$ \\
\hline Laberintitis & $\begin{array}{l}\text { Complicación } \\
\text { Otitis media aguda/crónica }\end{array}$ & Días & Hipoacusia unilateral \\
\hline Ototóxicos & Toma de ototóxicos & Lenta evolución & $\begin{array}{l}\text { Desequilibrio bilateral } \\
\text { Hipoacusia }\end{array}$ \\
\hline Traumatismos & $\begin{array}{l}\text { Trauma } \\
\text { Barotrauma }\end{array}$ & $\begin{array}{l}\text { Minutos } \\
\text { Días }\end{array}$ & $\begin{array}{l}\text { Hipoacusia } \\
\text { Acúfeno }\end{array}$ \\
\hline \multicolumn{4}{|l|}{ Centrales } \\
\hline Migrañoso & Migraña (aura visual, fotofobia) & Minutos, horas, días & $\begin{array}{l}\text { Manifestaciones centrales } \\
\text { o periféricas }\end{array}$ \\
\hline Accidente isquémico transitorio & Factores de riesgo vascular & Minutos a horas & Sintomatología neurológica focal \\
\hline Infarto y hemorragia cerebelosa & Factores de riesgo vascular & Días o semanas & Ataxia \\
\hline Esclerosis múltiple & Enfermedad degenerativa & Días o semanas & $\begin{array}{l}\text { Neuritis retro bulbar, diplopía, } \\
\text { paresias y parestesias }\end{array}$ \\
\hline
\end{tabular}


An Med (Mex) 2019; 64 (4): 290-296

Tabla 2: Características distintivas del vértigo periférico y central.

\begin{tabular}{lll}
\hline Clínica & Periférico & Central \\
\hline Náusea y vómito & Severo & Moderado \\
Nistagmo & Horizontorrotatorio o rotatorio & Vertical (cualquier dirección) \\
Fijación & Suprime a la fijación & No suprime con la fijación \\
Fatigable & Menos intenso cuando se repiten las pruebas & Nistagmo mantiene la intensidad \\
Intensidad & Sigue la ley de Alexander & No cambia la intensidad \\
Romberg & Cae de lado contrario de la fase rápida del nistagmo & Cae del mismo lado \\
Hipoacusia asociada & Común & Raro \\
Maniobra Dix-Hallpike & Nistagmo rotatorio hacia el oído dependiente: disminuye en & Nistagmo en varios patrones. Persistente \\
& aproximadamente 50 segundos & \\
Latencia & Los síntomas inician 1-5 segundos o después de la maniobra & Síntomas inician en conjunto con la maniobra \\
Extinción & Se agota tras varias maniobras & Nunca se agota \\
\hline
\end{tabular}

fálico, marcha inestable y nistagmo, el cual dura de horas a semanas. ${ }^{3}$

\section{Enfermedades del sistema vestibular periférico}

Las enfermedades del sistema vestibular periférico se limitan al VIII nervio craneal y sus estructuras distales. La ilusión de rotación está altamente relacionada con lesiones periféricas, los pacientes que las presentan generalmente tienen nistagmo horizontorrotatorio, que se suprime con la fijación visual. El nistagmo empeora con la mirada opuesta al sitio de la lesión. ${ }^{5}$ Los síntomas vegetativos son frecuentes, dentro de los cuales se hallan náusea, vómito, diaforesis y bradicardia. La tasa de recuperación disminuye con la edad y con la severidad del caso.

En la exploración neurológica, es necesario revisar la agudeza visual, los campos visuales y los movimientos oculares; asimismo, se debe realizar la exploración del reflejo oculomotor, rastreo y convergencia. El examinador debe enfocarse en los movimientos oculares y la posición de la mirada y buscar nistagmo en reposo o posicional. ${ }^{9} \mathrm{Si}$ se encuentra nistagmo espontáneo, nos habla de un desbalance entre el sistema vestibular central y periférico.

La prueba de impulso cefálico horizontal (head thrust test), descrito en 1988 por Hylmagy y Curthoys, ${ }^{9}$ es una herramienta rápida, sencilla y de fácil interpretación, que se utiliza para valorar el reflejo óculo-vestibular, y así detectar la presencia de vestibulopatías uni o bilaterales en la cabecera del paciente. Para realizarlo, el paciente se sienta enfrente del examinador, se pide que fije la mirada en un punto estático (nariz del examinador) y se mueve rápidamente la cabeza del paciente $10 \mathrm{o} 15^{\circ}$ hacia un lado, buscando sacada correctiva de los ojos. Se repite la misma maniobra en el sentido opuesto. La alteración del reflejo óculo-vestibular la observamos cuando se observa un retraso en el movimiento de los ojos y hay una sacada de reajuste. ${ }^{1,9,11}$

La prueba de desviación oblicua de la mirada se trata de una desalineación vertical de los ojos, que resulta de un desbalance del tono vestibular derechaizquierda. ${ }^{9,11}$ Se considera un signo bastante específico de patología central, sobre todo por la existencia de infarto en el bulbo lateral o la protuberancia.

En el año 2009, se propuso combinar estas tres exploraciones, llamándolas Head Impulse, Nystagmus, and Test of Skew (HINTS, por sus siglas en ingles). En manos de especialistas, esta prueba tiene una sensibilidad de $99 \%$ y una especificidad de $97 \%$ en identificar causas de origen central, ${ }^{8}$ mientras que la resonancia magnética $(\mathrm{RM})$, de forma inmediata en un paciente con vértigo, no es suficiente para excluir el diagnóstico de un evento cerebrovascular. ${ }^{9}$

Algunos estudios no aceptan como diagnóstico fiable una resonancia magnética $(\mathrm{RM})$ negativa en un cuadro de menos de 72 horas si los signos oculomotores sugieren otra cosa. Esto es de suma importancia, ya que la tasa de diagnóstico incorrecto de ictus en fosa posterior, cuya forma de presentación es vértigo, es hasta de $35 \% .^{6,12,13}$

\section{Vértigo postural paroxístico benigno}

El vértigo postural paroxístico benigno (VPPB) se considera la causa más común de vértigo periférico. Es más frecuente en mujeres en la cuarta y quinta década de la vida. ${ }^{10}$

En 1980, Epley propuso la teoría de que densidades libres (otolitos) flotando dentro de los canales 
semicirculares eran los causantes de la sensación de vértigo. ${ }^{5} \mathrm{El}$ síntoma más frecuente es la afección al canal posterior (60-90\%), aunque se pueden involucrar el canal anterior y lateral. ${ }^{2}$

Los pacientes con VPPB se quejan de vértigo al cambio de posición, con un inicio abrupto de los síntomas y una duración breve, menor a un minuto. Los factores de riesgo para síntoma son: sexo femenino, depresión durante el último año, hipertensión, dislipidemia, diabetes mellitus, enfermedad coronaria, antecedente de evento cerebrovascular y sobrepeso. Un ataque clásico se caracteriza por nistagmo evocado por movimiento cefálico con una latencia menor a 20 segundos; existe una disminución de la intensidad y duración con la repetición de la maniobra.

El diagnóstico es clínico. Se realiza principalmente a partir de la historia clínica y la exploración física, evocando nistagmo con maniobra de Dix-Hallpike para el canal posterior, y roll test, para el lateral. La maniobra de Dix-Hallpike se considera el estándar de oro para el diagnóstico del VPPB del canal semicircular posterior, cuya sensibilidad se presenta de $82 \%$ y especificidad de $71 \% .^{10}$

La primera línea de tratamiento son las maniobras de reposicionamiento, con una resolución de los síntomas de $80 \%$ en la primera maniobra y de $92 \%$ en la segunda. Se realiza la maniobra de Epley cuando hay afección de canal semicircular posterior y la de Barbecue cuando hay afección del lateral. ${ }^{2}$

\section{Neuritis vestibular}

Es la segunda causa más común de vértigo de origen periférico. Se cree que se debe a una infección viral del nervio vestibular o isquemia laberíntica como causa de neuritis vestibular. En la actualidad, la teoría más aceptada es la infección viral, generalmente provocada por virus de la familia Herpesviridae. ${ }^{10,11}$ Se reporta una incidencia de infección de vías aéreas superiores previa a la neuritis vestibular de 23 a $100 \% .^{6,10}$

Se caracteriza por ser una crisis intensa de vértigo agudo espontáneo e incapacitante, con una duración de tres a seis días, así como inestabilidad y desequilibrio durante la marcha que pueden durar semanas, acompañado de intensa sintomatología vegetativa (náusea, vómito, taquicardia, y diaforesis). En este proceso, sólo hay afección de la porción vestibular del aparato vestibulococlear con ausencia de síntomas cocleares. ${ }^{6,13}$

En la exploración física, encontramos un nistagmo unidireccional espontáneo horizontorrotatorio, con fase rápida al lado sano, el cual disminuye a la fijación visual; se sugiere realizar la exploración con lentes de Frenzel. ${ }^{3}$ Hay una disminución de reflejo óculo-vestibular (head thrust) e hiporreflexia vestibular en pruebas calóricas. La audiometría y timpanometría se presentan sin alteraciones.

El tratamiento de inicio es de soporte, con antieméticos y frenadores laberínticos (recomendados por dos a tres días). Se han realizado múltiples estudios doble ciego, randomizados, donde no se ha demostrado ningún beneficio mediante el uso de antivirales (valaciclovir). Altas dosis de metilprednisolona han demostrado mejoría a corto y largo plazo de la función del nervio vestibular, aunque la recuperación generalmente es incompleta. ${ }^{6}$

\section{Enfermedad de Ménière}

Se trata de una patología vestibular periférica, la cual se caracteriza por crisis de vértigo que dura de minutos a horas, presentándose de forma episódica. Suele asociarse con hipoacusia neurosensorial, unilateral y fluctuante, y acúfeno constante o intermitente, el cual se exacerba antes o durante los ataques de vértigo y plenitud aural. ${ }^{6}$

Se cree que la patogénesis está asociada con un aumento de la presión del líquido endolinfático, pero la causa exacta del hidrops endolinfático se desconoce. ${ }^{5}$ Es más frecuente en pacientes caucásicos y mujeres y se presenta a los 20 y 40 años. ${ }^{5,6,10}$

El diagnóstico se establece por medio de la historia clínica, describiendo los episodios de vértigo, el acúfeno y la hipoacusia (previamente descritos). Se realizan pruebas de audiologías (audiometrías seriadas) y vestibulares (electronistagmografía y pruebas calóricas).

El tratamiento para un episodio agudo tiene el objetivo de controlar los síntomas, a través de antieméticos (metoclopramida, ondansetrón, clorpromazina) y sedantes menores (lorazepam, diazepam, difenhidramina, dimenhidrinato), que nos ayudan a disminuir el vértigo. ${ }^{2}$

El tratamiento para prevenir las recurrencias consiste en una dieta hiposódica (menos de $1 \mathrm{~g}$ de sodio al día), diuréticos como inhibidor de anhidrasa carbónica (acetazolamida), amilorida o hidroclorotiazida, y vasodilatadores como betahistina. Cuando éstos fallan, se consideran los tratamientos quirúrgicos, los cuales se clasifican en los que conservan o no la audición. Su elección se basa en los estudios audiométricos del paciente (con audición conservada, descompresión del saco endolinfático, neurectomía vestibular, shunt endolinfático) y para pacientes 
anacúsicos, aminoglucósidos intratimpánicos o con laberintectomía. $^{2}$

\section{Fístula perilinfática}

Resulta por una comunicación anormal entre el espacio perilinfático y el oído medio o por una comunicación intramembranosa. ${ }^{3}$ Pueden ser secundarias a procedimientos quirúrgicos como estapedectomía, trauma o barotrauma. Clínicamente, se puede presentar con síntomas vestibulares o cocleares. Los pacientes se quejan de desequilibrio posterior al incremento de la presión de líquido cefalorraquídeo (signo de Hennebert) positivo. ${ }^{5}$

En la exploración física, la prueba de fístula se presenta positiva, la cual consiste en poder provocar vértigo y nistagmo con el cambio de presión en el oído medio, así como también el fenómeno de Tullio, que consiste en provocar vértigo y nistagmo con la exposición a un estímulo sonoro intenso (100-110 decibeles). Los signos de Romberg también se presentan positivos, lateralizando al lado afectado. Inicialmente el tratamiento es conservador con reposo, laxantes, elevación cefálica y audiometrías seriadas. ${ }^{2,3}$ La exploración quirúrgica se sugiere para la identificación del sitio de fístula (exitosa en $50 \%$ de los casos). Dependiendo el estudio, cuando se logra cerrar la fístula, los síntomas vestibulares mejoran de 83 a $94 \%$ y los auditivos de 13 a $49 \%$.

\section{Enfermedades del sistema vestibular central}

\section{Migraña vestibular}

Considerado como un síndrome vascular, resulta de la dilatación y la vasoconstricción serial de vasos intracraneales. La migraña se define, según la International Head Society, como cefalea intermitente, recurrente, unilateral, pulsátil y discapacitante, que está asociada con náuseas, fonofobia y fotofobia, con o sin aura (síntomas focales neurológicos). ${ }^{5}$ Treinta y ocho por ciento de los pacientes con migraña presentan episodios de vértigo; $1 \%$ de la población sufre de esta entidad, siendo más frecuente en mujeres (2:1). Su incidencia ocurre en la edad productiva, durante la tercera y cuarta década de la vida. ${ }^{14}$

La migraña vestibular se define como todo vértigo o mareo con duración de cinco minutos a 24 horas, causado o relacionado con la migraña o con el complejo migrañoso. Ésta se describió por primera vez en
1861; Prosper Ménierè mencionó que tenían síntomas en común. El diagnóstico es complejo y requiere cumplir los criterios según la International Classification of Headache Disorders (ICHD-II, por sus siglas en inglés).

\section{Migraña vestibular definida:}

a. Síntomas vestibulares episódicos, que deben ser al menos moderados o severos en intensidad.

b. Historia actual o previa de migraña de acuerdo con el ICHD-II.

c. Uno de los siguientes síntomas migrañosos durante dos o más ataques de vértigo: dolor de cabeza migrañoso, fotofobia, fonofobia, aura visual $u$ otro tipo de aura.

d. Otras causas que hayan sido descartadas por estudios apropiados.

\section{Migraña vestibular probable:}

a. Síntomas vestibulares episódicos que deben ser al menos moderados o severos en intensidad.

b. Uno de los siguientes:

Historia actual o previa de migraña de acuerdo con el ICHD-II.

- Síntomas migrañosos durante los síntomas vestibulares.

- Precipitantes de migraña en más de $50 \%$ de los ataques de vértigo (gatillos alimentarios, irregularidades del sueño o cambios hormonales).

- Respuesta efectiva a la medicación antimigrañosa en más de $50 \%$ de los ataques.

c. Otras causas que hayan sido descartadas por los estudios apropiados (Tabla 3).

El tratamiento inicial es no farmacológico; se trata pues de modificaciones en el estilo de vida: evitar estrés, deshidratación, ayuno prolongado, evitar agentes gatillo como alimentos (chocolate, vi-

Tabla 3: Síntomas de migraña vestibular.

\begin{tabular}{ll}
\hline Síntomas vestibulares & \multicolumn{1}{c}{ Síntomas auditivos } \\
\hline $70 \%$ vértigo rotacional & $66 \%$ fonofobia \\
$48 \%$ intolerancia a movimientos cefálicos & $63 \%$ acúfeno \\
$22 \%$ vértigo posicional & $32 \%$ hipoacusia \\
& $11 \%$ hipoacusia fluctuante + \\
& plenitud aural \\
\hline
\end{tabular}


nos, quesos, etcétera), además de que se recomienda sueño y ejercicio. En pacientes donde falla, se recomiendan fármacos abortivos o para controlar el episodio agudo como dimenhidrinato, metoclopramida, y analgésicos comunes (naproxeno o paracetamol) o ergotamina y triptanos (zolmitriptán, sumatriptán). Existen también tratamientos profilácticos como benzodiacepinas, bloqueadores de canales de calcio, beta-bloqueadores, inhibidores selectivos de la recaptura de serotonina, entre otros. ${ }^{2}$

\section{Infarto vertebrobasilar}

La irrigación al tronco cerebral, cerebelo y oído interno está dada por el sistema vertebrobasilar, por lo que cualquier oclusión en sus ramas principales podría ser síntoma vestibular. El vértigo es de los primeros síntomas en los infartos de la fosa posterior. ${ }^{5}$ Los síntomas de un evento isquémico vertebrobasilar es muy variable, dependiendo de la rama afectada, ya sea la arteria cerebelar posteroinferior, anteroinferior o cerebelar superior. Generalmente, en las lesiones centrales, el nistagmo espontáneo no se suprime con la fijación de la mirada. ${ }^{7}$ Múltiples procesos pueden ocluirlas: ateroesclerosis, émbolos o disección vascular secundaria a trauma o manipulación. ${ }^{10}$

La oclusión de la arteria cerebelar posteroinferior presenta síndrome de Wallenberg, el cual incluye vértigo, nistagmo, alteraciones de la marcha, ataxia distal ipsilateral, dolor o parestesias faciales ipsilateral y hemianestesia corporal contralateral, síndrome de Horner, disfagia, disfonía y disartria. ${ }^{7}$

El infarto pontomedular lateral, secundario a la oclusión de la arteria cerebelar anteroinferior, manifiesta síndrome pontino inferior, caracterizado por el involucramiento del VII y VIII nervio craneal, con parálisis facial ipsilateral, acúfeno e hipoacusia ipsilateral.

El síndrome pontino superolateral secundario a la oclusión de la arteria cerebelar superior muestra vértigo, nistagmo, alteraciones de la marcha, ataxia distal ipsilateral, dolor o parestesias faciales ipsilaterales, hemianestesia corporal contralateral y síndrome de Horner. Se distingue porque se presenta alteración de la temperatura y la vibración por afección del lemnisco medial. ${ }^{15}$

Se debe de tener un alto índice de sospecha en pacientes que inician con vértigo espontáneo y cualquier otro signo o síntoma neurológico concomitante, para evitar una falla en el diagnóstico y tratamiento.

\section{Insuficiencia vertebrobasilar o ataque isquémico transitorio (AIT)}

Tiene síntomas similares a los del infarto vertebrobasilar, pero éstos remiten a las 24 horas. Si no se da tratamiento, la enfermedad progresa a un infarto con secuelas. Veintinueve por ciento de los pacientes sufren como mínimo un episodio de vértigo previo al infarto vertebrobasilar. Es una causa común de vértigo en la tercera edad. En un tercio de los pacientes, el vértigo es la única manifestación de la enfermedad. Se debe tener en mente como un diagnóstico diferencial en pacientes con vértigo. Se presenta como ataques recurrentes y espontáneos de vértigo, los cuales incrementan en frecuencia. ${ }^{3}$

En pacientes en los cuales se presenten alteraciones visuales o del estado de alerta, se debe realizar una evaluación urgente con protocolo de infarto cerebral e interconsulta con Neurología. ${ }^{15}$

\section{CONCLUSIÓN}

El vértigo es un síntoma con múltiples causas; para determinar su etiología es importante enfocarse en realizar una historia clínica detallada, determinando su duración, desencadenantes y síntomas asociados. El primer paso en la evaluación del paciente es situarlo en una de las categorías más específicas: vértigo, desequilibrio, presíncope o mareos inespecíficos. La exploración física y estudios complementarios nos ayudan a determinar si la patología es de origen central o periférico.

En los casos de vértigo central, asociado con signos neurológicos, es necesario derivar a nuestro paciente al servicio correspondiente (Neurología).

\section{BIBLIOGRAFÍA}

1. Kerber KA, Baloh RW. The evaluation of a patient with dizziness. Neurol Clin Pract. 2011; 1 (1): 24-33.

2. Post RE, Dickerson LM. Dizziness: a diagnostic approach. Am Fam Physician. 2010; 82 (4): 361-368.

3 . Kerber KA. Vertigo and dizziness in the emergency department. Emerg Med Clin North Am. 2009; 27 (1): 39-50, viii.

4. Spiegel R, Kirsch M, Rosin C, Rust H, Baumann T, Sutter R et al. Dizziness in the emergency department: an update on diagnosis. Swiss Med Wkly. 2017; 147: w14565.

5. Labuguen RH. Initial evaluation of vertigo. Am Fam Physician. 2006; 73 (2): 244-251.

6. Kerber KA, Brown DL, Lisabeth LD, Smith MA, Morgenstern LB. Stroke among patients with dizziness, vertigo, and imbalance in the emergency department: a population-based study. Stroke. 2006; 37 (10): 2484-2487. 
7. Tusa RJ, Gore R. Dizziness and vertigo: emergencies and management. Neurol Clin. 2012; 30 (1): 61-74, vii-viii.

8. Saber-Tehrani AS, Kattah JC, Mantokoudis G, Pula JH, Nair D, Blitz A et al. Small strokes causing severe vertigo: frequency of false-negative MRIs and nonlacunar mechanisms. Neurology. 2014; 83 (2): 169-173.

9. Kattah JC, Talkad AV, Wang DZ, Hsieh YH, Newman-Toker DE. HINTS to diagnose stroke in the acute vestibular syndrome: threestep bedside oculomotor examination more sensitive than early MRI diffusion-weighted imaging. Stroke. 2009; 40 (11): 3504-3510.

10. Thompson TL, Amedee R. Vertigo: a review of common peripheral and central vestibular disorders. Ochsner J. 2009; 9 (1): $20-26$.
11. Breinbauer KHA, Anabalón BJL. Prueba de impulso cefálico. Rev Otorrinolaringol Cir Cabeza Cuello. 2011; 71 (2): 123-130.

12. Alcalá-Villalón T, Lambert-García M, Suárez-Landrean A. Enfoque clínico del vértigo desde la Atención Primaria de Salud. Rev Haban Cienc Méd. 2014; 13 (3): 394-405.

13. Chan Y. Differential diagnosis of dizziness. Curr Opin Otolaryngol Head Neck Surg. 2009; 17 (3): 200-203.

14. Bittar RS, Lins EM. Clinical characteristics of patients with persistent postural-perceptual dizziness. Braz J Otorhinolaryngol. 2015; 81 (3): 276-282.

15. Karatas M. Central vertigo and dizziness: epidemiology, differential diagnosis, and common causes. Neurologist. 2008; 14 (6): 355-364. 